$\mathbb{T}$ periodica polytechnica

\author{
Mechanical Engineering \\ $52 / 2(2008) 103+106$ \\ doi: 10.3311/pp.me.2008-2.10 \\ web: http://www.pp.bme.hu/me \\ (c) Periodica Polytechnica 2008 \\ RESEARCH ARTICLE \\ János Viczai \\ Received 2008-10-17
}

\section{Geothermal energy mining}

\begin{abstract}
In Hungary the geothermal gradient is relatively high which means that there are high-temperature rocks underground. In theory the extraction of the heat of the rocks would be a perfect solution to ensure the heating of the country. The mathematical model written in the essay demonstrates that the exothermic system - consisted of two wells - is operable. The re-pressing was proved to be highly possible by many measurements, so the technological solution is ensured. In the USA scientists developed the hot dry stone method, when they make path for the fluid into the stones. Different system is used in France, when they repumping the fluid into the leaky stone.
\end{abstract}

\section{Keywords}

Geothermal heating

\section{János Viczai}

Department of Energetics and Service Systems, BME, H-1111 Budapest, Múegyetem rkp. 3. II. 45., Hungary

e-mail: viczai@egt.bme.hu

\section{The origin of the geothermal energy}

Approaching the depth of earth the temperature grows continuously. The rise in temperature was already noticed in the $17^{\text {th }}$ century on the basis of the measurements taken in different mines and observations of English and French scholars. For the sake of the exploration of the water and the hydrocarbon, in the last century the exploration work gradually developed the technology of the deep-drilling that is why deeper drills could take place. In the depth of more than thousand meters they not only fund water, oil and gas, but they also ascertained the existence of the geothermal energy, its physical parameters and they examined their conformation in the function of different geological, hydrogeological conditions.

The presence of the geothermal energy in every points of the earth can be ascertained but its extent is significantly influenced by local conditions. Therefore approaching the depth the measure of the warming is territorially changing. The index number of this warming's value called temperature gradient $\left({ }^{\circ} \mathrm{C} / \mathrm{km}\right.$ or ${ }^{\circ} \mathrm{C} / \mathrm{m}$ ), which shows how much ${ }^{\circ} \mathrm{C}$ rise in temperature belongs to the accreation of $1 \mathrm{~km}$ (or $\mathrm{m}$ ) depth in the examined area. In the practice we use invent of the gradient (Fig. 11. In world average it is $30^{\circ} \mathrm{C}$ measured on earth radius but in some territory it is higher and for instance it is $50-60^{\circ} \mathrm{C}$. The value is higher in the post vulcanic areas.

The origin of the geothermal energy can be attributed to the processes of the atomic fission, in which processes the earth functions as an atomic furnace and at this process the heat flows towards the surface. This ground heatflux's index number is 62 $\mathrm{mWm}^{-2}$ in average. In Hungary it is higher, $84 \mathrm{mWm}^{-2}$. We can say that this value is extremely low nevertheless it is sufficient to warm up the solid rock-mass very slowly. Practically the present geothermal energy production exploits these accumulated heat energy.

\section{The method of the usage}

However this accumulated heat energy can only be brought to surface from hot solid rocks with the help of a heat carrier. Up to this point it was only possible where in greater depth different liquid or gaseous substances take place, which absorbs 


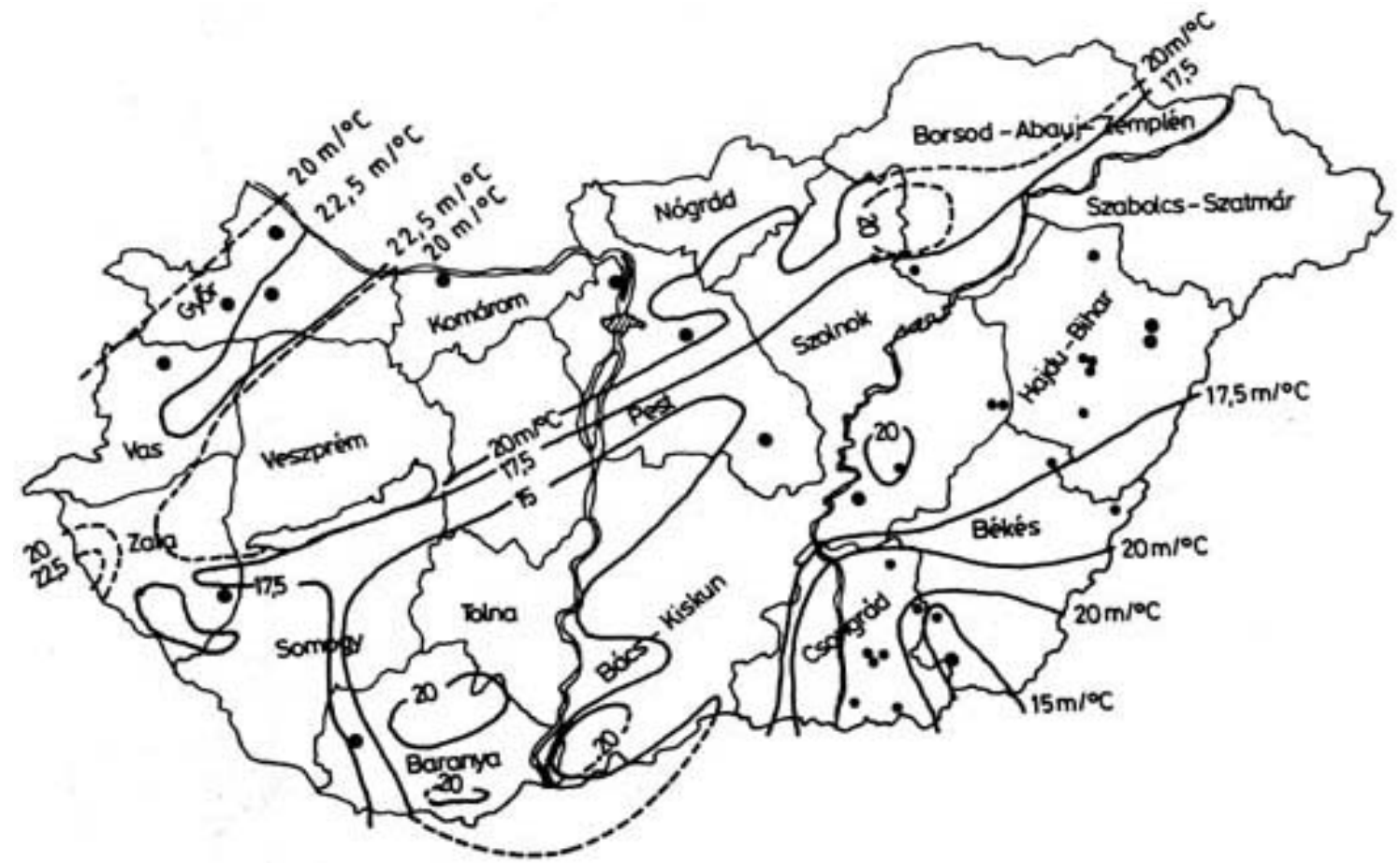

Fig. 1. The geothermal gradient in Hungary

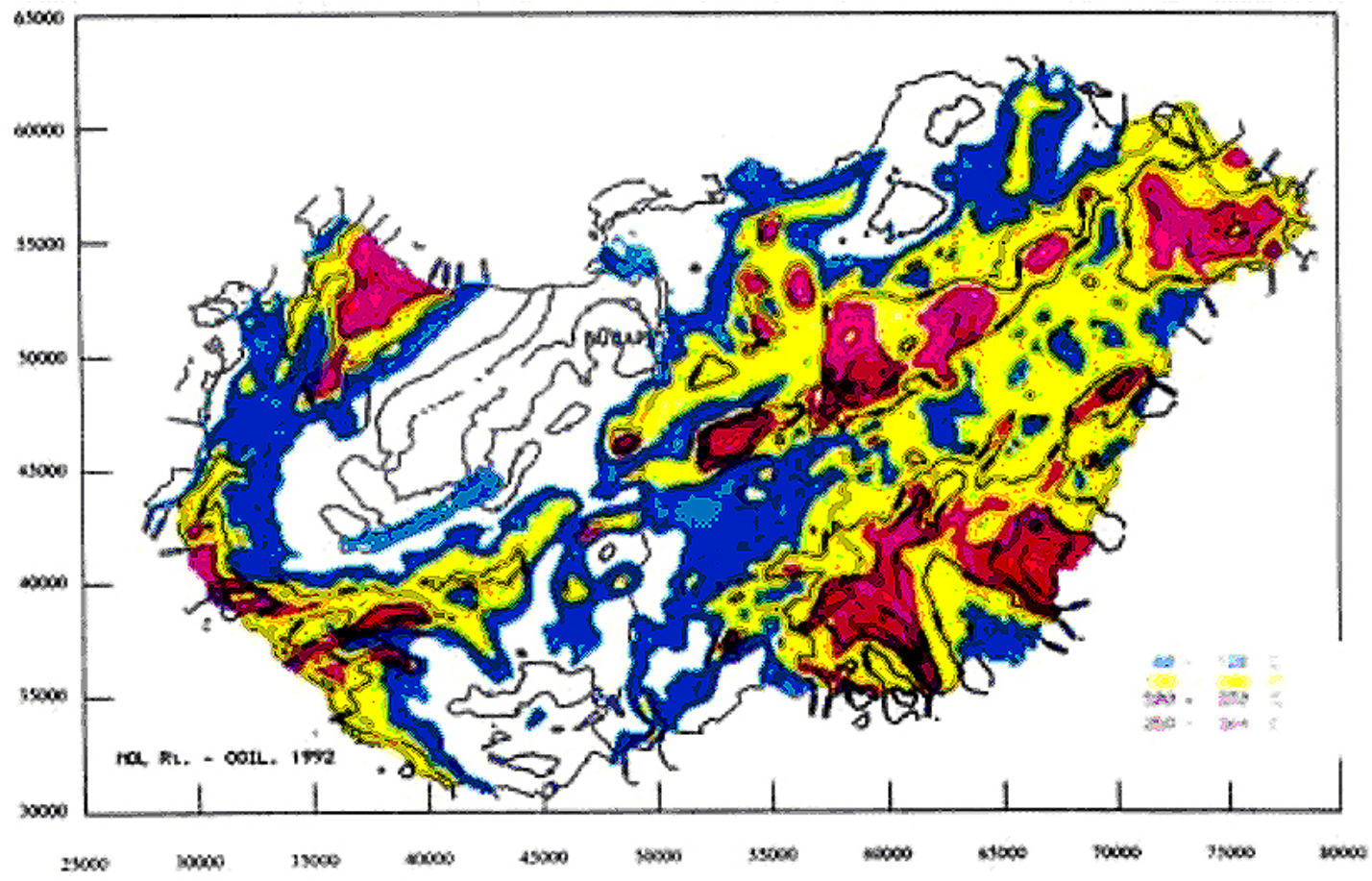

Fig. 2. The basicmountain temperature 
the temperature of the environment. The heat carrier could have appeared as a geyser, thermal water in a natural way of deepdrillings. These drillings can be different gases, oils but most frequently the water - being in different state - fills the part of the heat carrier. In the depth, the water takes place in the porous rocks, cracks and getting to surface being depended on its temperature, it presents in the form of 100 Celsius thermal-water. If there is no heat carrier medium, with the help of a producer and a pressing pair of wells can we exploit heat. As in Hungary more than one thousand prospector wells exist therefore we can develop this kind of system in almost every borders of any settlements.

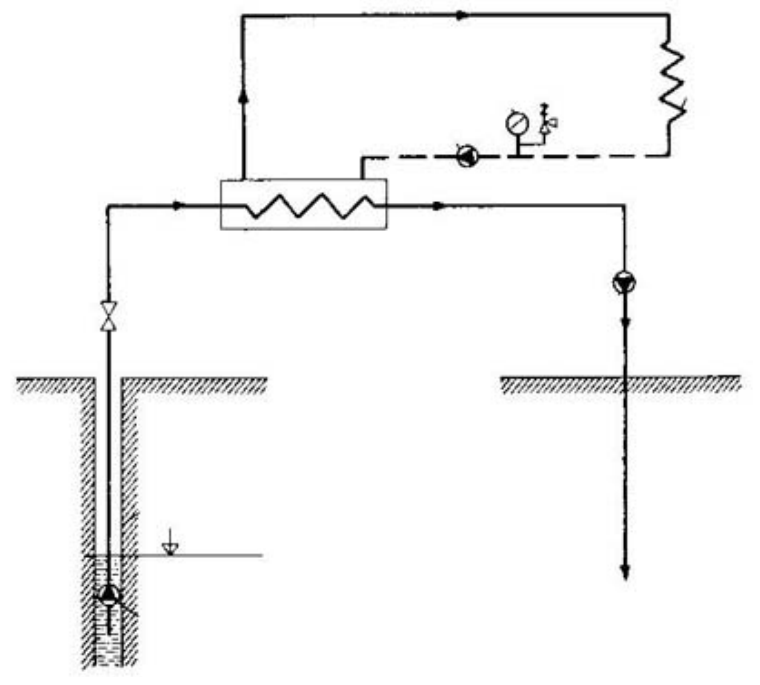

Fig. 3. Two wells system model

\section{The mathematical model}

After that we have to examine that one producer and repressing pair of wells how we storage heater medium affect the geothermal and hydrogeological's relation. The selected area is average 20-25 meter thick Upper-Pannon sandstone (700 mD) with good permeability. Minimal hydraulic gradients to be developing regional size flow systems in this good permeability layer. The thickness of layer is in negligible next on horizontal extent. As follows the leaking water is available two dimensions with good approach.

The flowing layerwater is uncompressible, the developing velocity field is rotary free. In the vertical level the linear partial equation is describable with the XY linear and orthogonal coordinate system and the $\mathrm{v}_{x}, \mathrm{v}_{y}$ components of leaking velocity:

$$
\begin{aligned}
& \frac{\partial v_{x}}{\partial x}+\frac{\partial v_{y}}{\partial y}=0 \\
& \frac{\partial v_{y}}{\partial x}+\frac{\partial v_{x}}{\partial y}=0
\end{aligned}
$$

The first equation means the uncompressibility, the second equation means the rotary free. If these equations to be realized in every points of the layer, to be exist two scalar equation which satisfy the LAPLACE criteria and one another harmonic pairs. $\mathrm{v}_{x}, \mathrm{v}_{y}$ velocity components are represent with these $\mathrm{F}_{1}$ and $\mathrm{F}_{2}$ function:

$$
\begin{aligned}
& v_{x}=\frac{\partial F_{1}}{\partial x}=\frac{\partial F_{2}}{\partial y} \\
& v_{y}=\frac{\partial F_{1}}{\partial y}=\frac{\partial F_{2}}{\partial x}
\end{aligned}
$$

The third and the fourth equation are the CAUCHYRIEMANN differential equation-pair. These have got infinite solutions. The flow complex potential is:

$$
P=F_{1}(x, y)+i \cdot F_{2}(x, y)
$$

This is a regular complex variable function, and its real part the $F_{1}$ velocity potential, $F_{2}$ flow image is the imaginary part. The leaking velocity's conjugal is the $P$ function $z$ numeral derivation:

$$
\bar{v}(z)=\frac{d P}{d z}=v_{x}-i \cdot v_{y}
$$

In the knowledge of these equations any parameters are calculable. The Fig. 4 shows the position of the wells. The wells' distance is $2 \cdot d$, the pressing wells label is $-m$, the re-pressing wells label is $+m$.

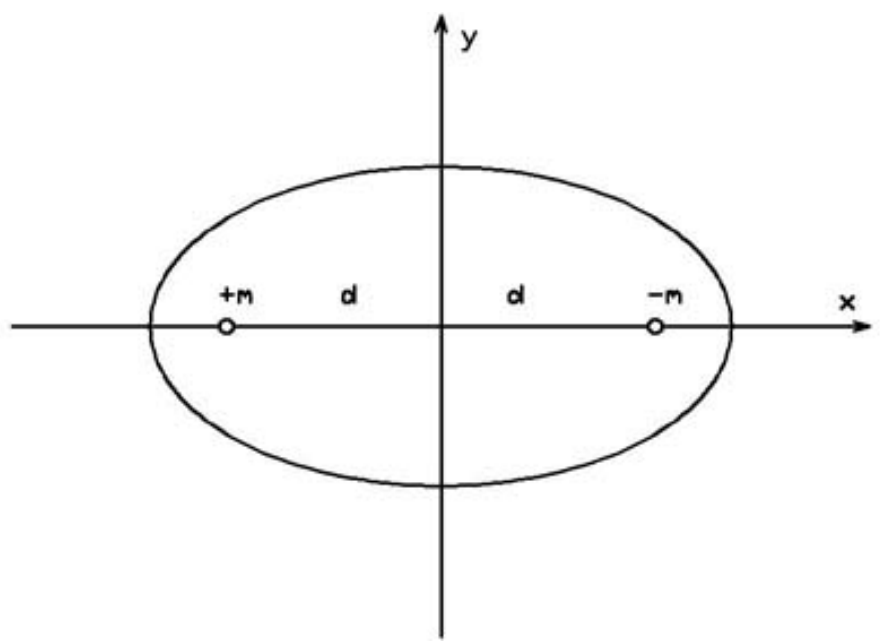

Fig. 4. The wells' position

The $v_{0 x}$ velocity's direction is same the $x$ axis direction. It is the undisturbed and the shortest flow picture. The complex potential's function:

$$
P=v_{0 x} \cdot z+\frac{m}{2 \cdot \pi} \cdot \ln (z+d)-\frac{m}{2 \cdot \pi} \cdot \ln (z-d)
$$

Near the wells the velocity field is:

$$
\bar{v}=v_{0 x}+\frac{m}{2 \cdot \pi}\left[\frac{1}{z+d}-\frac{1}{z-d}\right]
$$

After simplification:

$v_{x}=v_{0 x}+\frac{m}{2 \cdot \pi}\left[\frac{1}{z+d}-\frac{1}{z-d}\right]=v_{0 x}+\frac{m}{2 \cdot \pi} \cdot \frac{d}{d^{2}-x^{2}}$ 
The minimum of velocity is:

$$
v_{\min }=v_{0 x}+\frac{m}{d \cdot \pi}
$$

The average of velocity is:

$$
v_{a v}=\frac{1}{2 \pi} \int_{-d}^{+d}\left(v_{0 x}+\frac{m}{2 \cdot \pi} \cdot \frac{d}{d^{2}-x^{2}}\right) d x
$$

The average time while the water circulated once is:

$$
y_{a v}=\frac{2 \cdot d}{v_{a v}}
$$

The energy balance is:

$$
\begin{aligned}
& \int_{V} \frac{\partial}{\partial t}\left[(1-p) \cdot \varsigma_{r} \cdot c_{r} \cdot T_{r}+p \cdot \varsigma_{w} \cdot c_{w} \cdot T_{w}\right] d V= \\
& =\int_{A}\left[(1-p) \cdot \lambda_{r}+p \cdot \lambda_{w}\right] \operatorname{grad} T \overline{d A}
\end{aligned}
$$

$p$ - porosity, $c_{r}$ - specific heat of rock, $c_{w}-$ specific heat of water, $T$ - temperature, $\varsigma$ - density, $\lambda$ - thermal conductivity.

The heat energy from the pressing well is:

$$
E_{p}=\varsigma_{w p} \cdot c_{w} \cdot t_{w p} \cdot m
$$

The heat energy into the re-pressing well is:

$$
E_{r}=\varsigma_{w r} \cdot c_{w} \cdot t_{w r} \cdot m
$$

The gain heat energy is:

$$
E_{\text {gain }}=E_{p}-E_{r}
$$

\section{Summary}

The gain heat energy is $2-3 \cdot 10^{11} \mathrm{~kJ} / \mathrm{year}$ if the average time while the water circulated once is $0.8-1.3$ year and $m=1$ million $\mathrm{m}^{3} /$ year and $t_{v p}-t_{v r}=50-60{ }^{\circ} \mathrm{C}$. The re-pressing was proved to be highly possible by many measurements, so the technological solution is ensured. This mathematical model works individual wells and many wells system. The gain is positive, and with the clean electrical energy from wind turbines, we got really clean heat energy for the buildings.

\section{References}

Gróf Gy, Hôvezetés, 1998. BME discourse note.

2 Eisler Z, Közönséges differenciálegyenlek a gyakorlatban, 2002. BME discourse note.

3 Kontra J, A geotermális energia exergia szintú elemzése, Debreceni Múszaki Közlemények 5/3 (2006), 5-12.

$4 \_$, Role of geothermal energy in district heat supply in Hungary, Periodica Polytechnica Civil Eng. 51/1 (2007), 21-24.

5 Blair S C, Permeability of Ironton-Galesville sandstone at elevated temperature and pressure, Enerstock, 1985.

6 Boisdet A, A. Menjoz A, Projet de stockage termique en aquifere profond, Enerstock, 1985.

\section{Appendix}

$\mathrm{x}, \mathrm{y}$ - linear and orthogonal coordinate system

$\mathrm{v}_{x}, \mathrm{v}_{y}-$ components of leaking velocity

$\mathrm{F}_{1} \quad$ - velocity potential

$\mathrm{F}_{2}$ - flow image

d - wells' distance

m - mass flow

$\mathrm{y}_{a v} \quad$ - average time

p - porosity

$\mathrm{c}_{r} \quad-$ specific heat of rock

$\mathrm{c}_{w} \quad-$ specific heat of water

$\mathrm{T}$ - temperature

$\varsigma-$ density

$\lambda-$ thermal conductivity

E - energy 